\title{
Investigation of Flow Rate on Chemical Bath Deposition of Silver Films Inside Hollow Polymer Cylinders
}

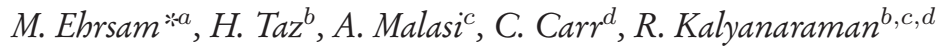 \\ ${ }^{a}$ Department of Mechanical, Aerospace and Biomedical, Engineering, University of Tennessee, Knoxville, TN. \\ ${ }^{b}$ Bredesen Center, University of Tennessee, Knoxville, TN. \\ ${ }^{c}$ Department of Chemical and Biomolecular Engineering, University of Tennessee, Knoxville, TN. \\ ${ }^{d}$ Department of Materials Science and Engineering, University of Tennessee, Knoxville, TN.
}

Students:mehrsam@vols.utk.edu*, htaz@vols.utk.edu, amalasi@vols.utk.edu, ccarr20@vols.utk.edu

Mentor:ramki@utk.edu

\begin{abstract}
In thin film solar cells made as planar structures, the absorption of solar light and hence, the solar conversion efficiency, is heavily determined by the position and orientation of the sun. One way to overcome this is to use solar cell geometries that could better absorb sunlight from different angles. One potential geometry is a hollow cylinder, which has the ability to better absorb the sun's rays incident at various angles due to the light trapping nature of the cylindrical geometry. If such solar cells could be fabricated inside polymer tubes with micrometer diameters, many potential applications built around non-woven or woven textiles could be realized. Here we investigate the deposition of homogeneous thin metallic films inside hollow polymer cylinders using the process of chemical bath deposition (CBD). Although films deposited via CBD have been studied extensively, mechanisms to achieve high quality deposition inside hollow cylinders are still not well understood. The objective of this project was to deposit a smooth, homogeneous silver film inside a hollow cylindrical structure using varying flow rates. The early stage film growth for very short deposition times was observed by the localized surface plasmon resonance of the silver nanoclusters via absorption spectra along the length of the tube. For longer deposition times, silver films formed and were analyzed for their morphology, thickness, roughness, and resistance using a combination of optical microscopy, scanning electron microscopy, and twoprobe conductivity. The findings from this study showed that deposition under flow with different Reynolds numbers had a strong influence on the morphology and electrical resistance of the deposited films.
\end{abstract}

\section{KEYWORDS}

Thin-films; Chemical Bath Deposition; Nanoparticles; Solar Cells; Silver

\section{INTRODUCTION}

Planar thin film semiconductors, which are the core of current generation solar cell technology, face fundamental challenges towards a continued reduction in cost while simultaneously increasing efficiency. For example, lower costs can be achieved by reducing the thickness of the semiconductor, eliminating materials, and reducing processing steps. However, ultrathin devices have significantly lower efficiency over bulk solar cell technology. For instance, Si ultrathin solar cell efficiencies are currently at $8-10 \%$, while that for bulk Si technology is at $15-25 \% .{ }^{1}$ The fundamental reason for this is the reduction in the amount of light trapped and absorbed in the ultrathin devices. A planar structure also has other disadvantages such as the efficiency and absorbency of the solar cell being heavily dependent on the position and orientation of the sun. Recently, a United States patent was awarded to a solar cell design in which the various metal and semiconductor layers of the solar cell were made by thin film deposition processes inside small diameter hollow cylinders, such as those made of polymer materials. ${ }^{2}$ The authors have suggested that the cylindrical geometry could better absorb the sun's rays due to its light trapping nature. In addition, the hollow nature of the substrate could also help in 


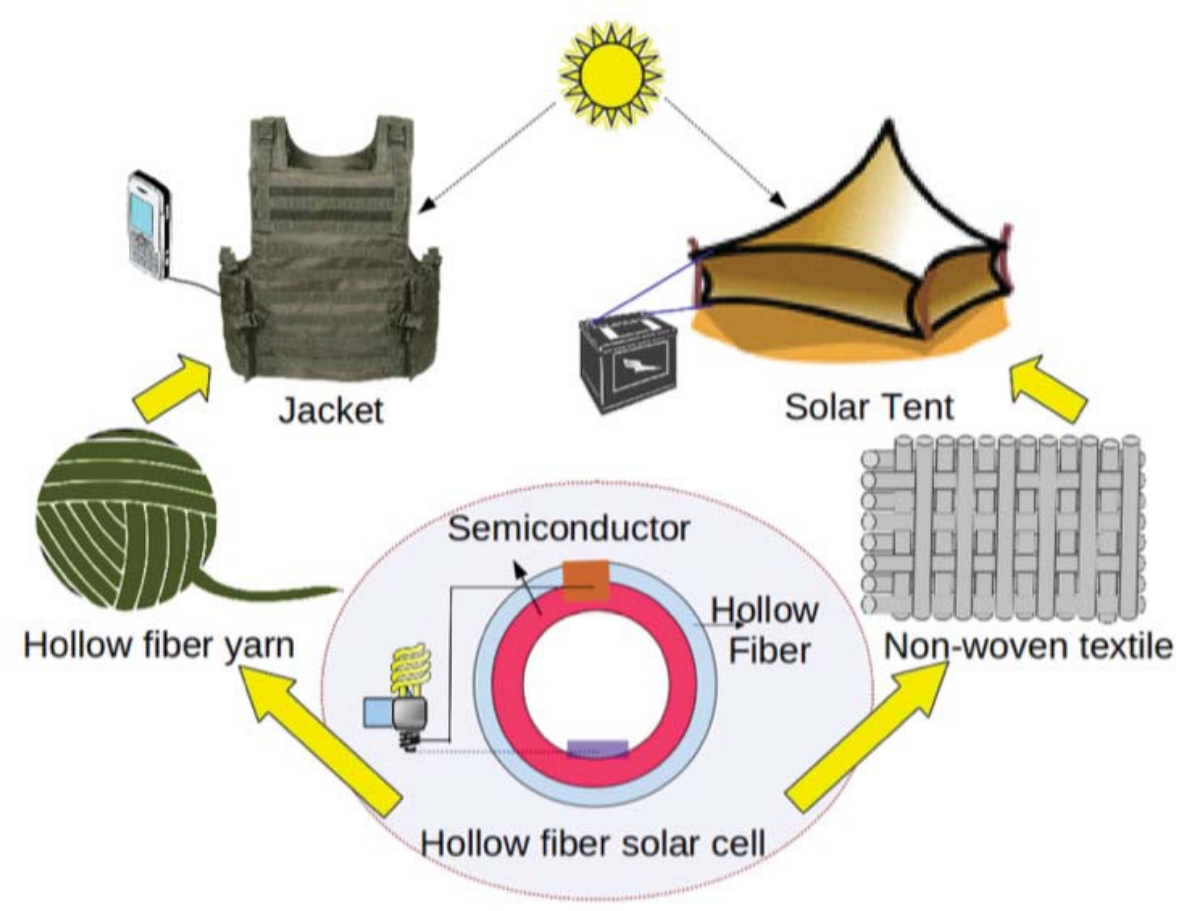

Figure 1. A schematic illustration of the potential applications that could be enabled by having a flexible hollow fiber solar cell. ${ }^{2}$

applications such as solar textiles as shown in Figure 1. Figure 1 depicts a semiconductor solar cell made inside a hollow polymer fiber. The vision is that this flexible hollow polymer fiber could be spun into a non-woven yarn or a woven fabric. Subsequently, the material can be transformed into a wearable device such as a jacket or a utilitarian device, such as a tent, from which solar energy can be harnessed.

A review of scientific literature has shown that there has been prior work on making organic and dye-sensitized solar cells on the outside of glass tubes or on fibers with a solid conducting core. ${ }^{3-7}$ Recently, the optical trapping properties of arrays of cylindrical radial junction solar cells motivated the fabrication of nanowire arrays of $\mathrm{Si}$ by various groups. ${ }^{8-12}$ However, there is little previous work on making inorganic solar cells inside hollow polymer fibers. Over the past decade, the chemical bath deposition (CBD) process has been successfully applied to deposit metal, semiconductor and dielectric films inside hollow fibers, largely for the purpose of infrared light transmission. ${ }^{13-25}$ In the CBD deposition process, salt solutions are mixed together at room temperature and flowed through the hollow fibers, resulting in a precipitation reaction of the desired metal, semiconductor or dielectric. ${ }^{26}$ The major focus of these previous works on deposition inside hollow fibers were on optimizing the film deposition conditions to obtain desired light transmission properties.

Here we present investigations of chemical bath deposition of silver $(\mathrm{Ag})$ metal films by the CBD process inside $22 \mathrm{~cm}$ long and $0.8 \mathrm{~cm}$ diameter hollow polymer tubes. Depending upon the rate at which a fluid flows through the tube, the resulting velocity profile of the fluid may be stationary (i.e. flow velocity of zero), laminar (smooth flow) or turbulent, and this could greatly impact the deposition kinetics of the thin films. Furthermore, when the fluid enters the tube, the velocity profile typically requires a characteristic distance before achieving its steady-state behavior, potentially leading to differences in deposition at the entrance versus further inside the hollow tube. Therefore, the goal of this work was to understand how the morphology of silver thin films were affected by the rate of flow as well as with distance from the entrance of the fluid under the CBD process. We have utilized a combination of scanning electron microscopy (SEM) imaging, electrical resistivity measurements, and optical spectroscopy of the Ag plasmon properties to understand the $\mathrm{CBD}$ deposition of $\mathrm{Ag}$. 


\section{METHODS AND PROCEDURES}

For the $\mathrm{CBD}$ of $\mathrm{Ag}$ thin films, we utilized the known process in which two solutions were required. ${ }^{24}$ The first was a silver precursor made of a $8.3 \%$ molar Silver Nitrate (Acros Silver Nitrate 99.85\%) solution that was titrated using ammonium hydroxide to form $2 \mathrm{Ag}\left(\mathrm{NH}_{3}\right)_{2} \mathrm{OH}$. Next, a $25 \%$ molar sodium hydroxide [Fisher Scientific Sodium Hydroxide (beads)] solution was added to this silver solution, and was titrated again with ammonium hydroxide. The second solution was a reducing agent: a 6.7\% molar aqueous solution of dextrose [Fisher Scientific Dextrose (D-Glucose) Anhydrous]. The densities of these solutions are shown in Table 1. These solutions were then pumped through a Tygon Tube (ID: $1 / 16$ in) into a $22 \mathrm{~cm}$ long commercially available poly propylene tube (D\&W Fine Pack) with a $0.8 \mathrm{~cm}$ diameter.

\begin{tabular}{|c|c|}
\hline Solution & Density $\left(\mathrm{g} / \mathrm{cm}^{3}\right)$ \\
\hline \hline Silver Nitrate Solution & 1.014 \\
\hline Sodium Hydroxide Solution & 1.007 \\
\hline Reducing Agent Solution & 1.000 \\
\hline Final Solution & 1.007 \\
\hline
\end{tabular}

Table 1. Densities of Experimental Solutions

\section{Deposition at varying flow rates}

For the first part of this experiment, $200 \mathrm{~mL}$ of the final solution was allowed to flow at four different flow speeds, identified as low, medium, high, highest. From Table 1, it can be seen that the densities of these solutions were very similar to that of water. Therefore, the flow rates and velocities of the pump were approximated by timing $100 \mathrm{~mL}$ of water flow through the $1 / 16$ in $(.0016 \mathrm{~m})$ Tygon tubes. The Reynolds Number was then calculated using the Reynolds number equation for flow in a pipe shown in Equation 1:27

$$
R e=\left(\rho v_{a v g} D\right) / \mu
$$

Equation 1.

where the average velocity $\left(v_{\text {avg }}\right)$ of each of the speeds is shown in Table 2, the dynamic viscosity $(\mu)$ of water is $0.00089 \mathrm{~Pa}^{*}$, the density of water at room temperature $(\rho)$ is $1000 \mathrm{~kg} / \mathrm{m}^{3}$, and the diameter of the tube $(\mathrm{D})$ is $\mathrm{D}=$ $0.0016 \mathrm{~m}$. Based on the calculation of the Reynolds number Re, presented in Table 2, all these speeds were in the laminar flow regime (i.e. $\operatorname{Re}<2600){ }^{27}$

\begin{tabular}{|c|c|c|c|c|}
\hline Pump Speed & Flow Rate $\left(\mathrm{m}^{3} / \mathrm{s}\right)$ & Velocity $(\mathrm{m} / \mathrm{s})$ & Reynolds Number $(\mathrm{Re})$ & Deposition time (s) \\
\hline \hline Low & $1.07 \times 10^{-7}$ & 0.053 & 95.4 & 1876 \\
\hline Medium & $1.91 \times 10^{-7}$ & 0.095 & 170.7 & 1048 \\
\hline High & $2.27 \times 10^{-7}$ & 0.113 & 203.3 & 880 \\
\hline Highest & $3.36 \times 10^{-7}$ & 0.167 & 300.2 & 596 \\
\hline
\end{tabular}

Table 2. Flow Parameters at Varying Speeds

The thickness and morphology of the deposited films were characterized at the entrance of the tube as well as at 10 and $20 \mathrm{~cm}$ distances along the tube using SEM (Zeiss Merlin). For this, the tubes were cut and samples were collected at the entrance of the tube and at the 10 and $20 \mathrm{~cm}$ positions. Prior to cutting the tubes, two-probe electrical conductivity (Keithley 2400) measurements were made for the total resistance of the deposited films along the entire length of the tube.

\section{Early stage deposition at highest speed}

For the second part of this experiment, the final solution was allowed to flow at the highest speed of $0.167 \mathrm{~m} / \mathrm{s}$ for 1 , 1.5, and 2 minutes. The localized surface plasmon resonance (LSPR) peaks of the deposited silver nanoparticles were analyzed at various locations along the length of the tube in $2 \mathrm{~cm}$ increments from 2 to $20 \mathrm{~cm}$, using optical spectroscopy (Ocean Optics UV-Vis Spectrometer). Additionally, the concentration of the silver nanoparticles at $1,1.5$ and 2 minutes were analyzed using SEM (Phenom ProX). 


\section{RESULTS AND DISCUSSION}

I. Deposition at Varying Flow Rates

a. Morphology: Figure 2 shows plane-view SEM micrographs depicting the contrast resulting from the deposited Ag films at the three different locations and for the various flow rates mentioned in Table 1. Based on these images, it was clear that the Ag film morphology appeared to depend both on position along the tube as well as the flow rate.

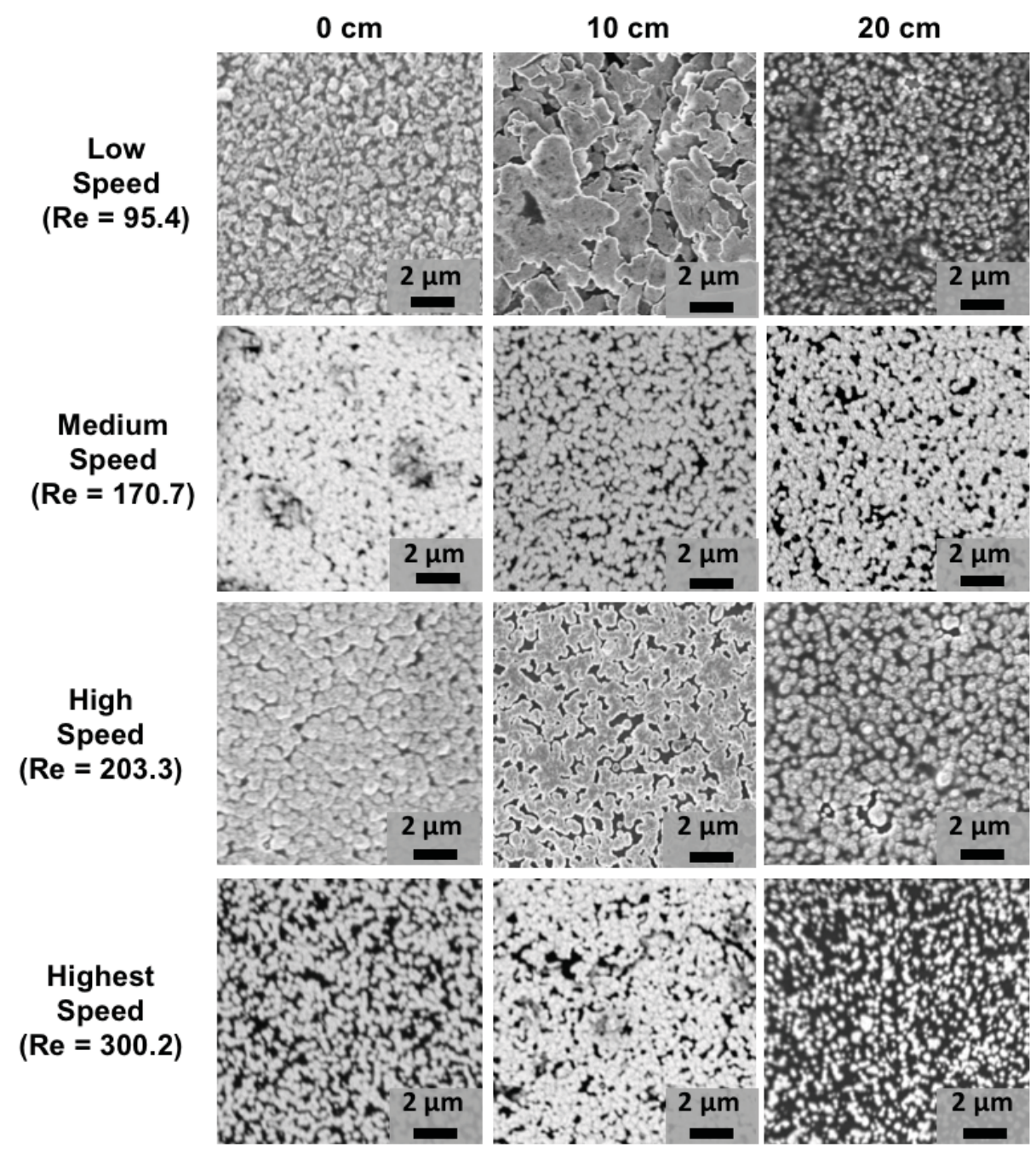

Figure 2. SEM images of deposited films at the Low, Medium, High, and Highest speeds (with the respective Re values indicated) at the entrance of the tube as well as 10 and $20 \mathrm{~cm}$ from the entrance.

For the low speed, there was a significant increase in the size of the Ag deposits in going from the entrance $(0 \mathrm{~cm})$ to $10 \mathrm{~cm}$, while the film appeared to consist of much finer features at $20 \mathrm{~cm}$. For the medium and high speeds, the film appeared to be fairly uniform along the length of the tube. It was also apparent that the microstructure became more aggregated or discontinuous towards the end of the tube. At the highest speed, the film appeared to have a smaller grained and discontinuous microstructure at the entry and end of the tube, with a more homogeneous deposit near the center (at $10 \mathrm{~cm})$.

b. Film thickness and deposition rate: After analyzing the plane-view morphology of the films, cross-sectional SEM was performed to analyze the thickness of the films for the four different speeds at the various locations (entrance $=0 \mathrm{~cm}$, middle $=10 \mathrm{~cm}$, near exit $=20 \mathrm{~cm}$ ). Figure 3(a) shows a typical cross-section of a Ag film deposited at low speed and 
taken at the entrance of the cylinder. The film thickness was estimated by averaging several thickness measurements (typically at least 5). Figure 3(b) plots the average thickness of the silver film at different locations of the tube as a function of the Reynolds number Re. The error bars for each measurement correspond to 1 standard deviation from the mean value.
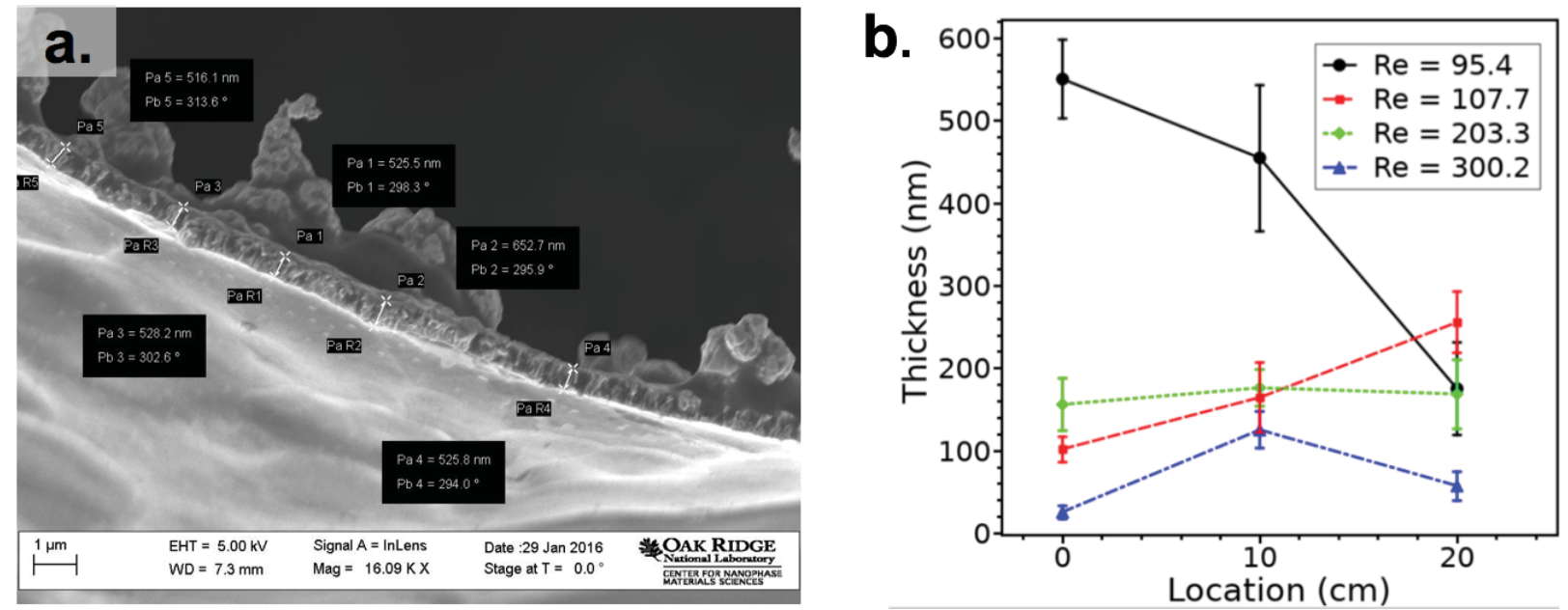

Figure 3. (a) Cross section SEM of silver film deposited at the low speed taken at the entrance of cylinder. (b) Thickness of film as a function of tube length.

In Figure 3(b), the thickness at each location was compared to the Reynolds number $R e$ for the various flow speeds. Near the entrance, the thickness dropped rapidly with increasing $R e$. In contrast to the low speed case with $R e=95$, the $R e=170$, showed an increase in film thickness across the length of the tube. The higher speed case of $R e=203$ showed a fairly uniform film thickness across the tube length. The highest speed (with $R e=300$ ) showed a much thinner film across the entire length as well as a larger thickness at the center of the tube $($ at $10 \mathrm{~cm})$ as compared to at the entrance and exit. For solar cell applications, it is important to have a homogeneous film thickness, and based on the thickness measurements in Figure 3(b), the high speed case with $R e=203$ appeared to be the most desirable for producing uniform thickness films. This suggested that for the conditions of our experiments, of tube diameter and flow rates, the $R e=203$ flow had the most optimal conditions of silver deposition along the inside walls of the tube.

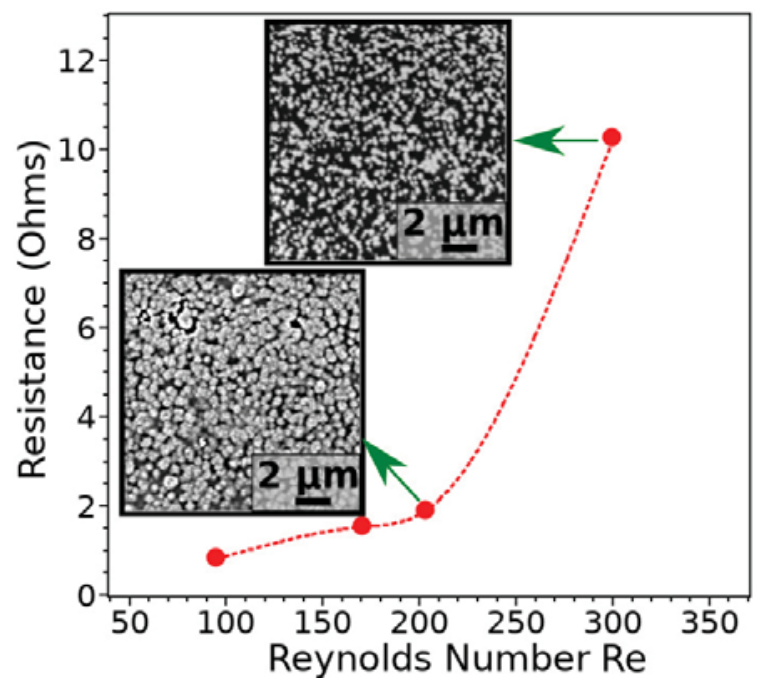

Figure 4. Measured resistance values of the deposited Ag films across the length of the hollow cylinder as a function of Re. SEM image of nanoparticles formed at $R e$ of 203 and 300 at the tube position of $10 \mathrm{~cm}$ are also shown. 
c. Film Resistance: In order to correlate the film thickness with film quality, the electrical resistance was measured by obtaining current versus voltage curves by placing electrical connections at either end of the tube so that the resulting behavior was representative of the entire length of the tube. From this data, the resistance versus Re (Reynolds number) was plotted and is shown in Figure 4. Based on these results, it is clear that for the films deposited with $95 \leq$ $R e \leq 203$, there was little change in overall film resistance. However, the highest speed with $R e=300$ resulted in nearly 5 fold increase in overall resistance across the tube. In order to understand why the resistance changed so significantly at the highest speed, the SEM images were analyzed. The insets in Figure 4 show the planar SEM images of the films at $\operatorname{Re}=203$ (high speed) and $\operatorname{Re}=300$ (highest speed). As mentioned earlier, with respect to Figure 3(b), the $R e=300$ case had the smallest thickness. Based on the SEM image shown in Figure 4, the microstructure for this case showed Ag deposit (bright regions) along with a large fraction of the dark regions, which corresponded to a lack of $\mathrm{Ag}$ deposit, suggesting poor connectivity between the Ag regions. In contrast, the SEM for the $R e=203$ case shown in Figure 4 indicated a much higher degree of connectivity between the bright regions corresponding to the Ag deposits. Therefore, the conclusion was that the higher resistance in the $R e=300$ case is a result of a poorly connected thin film morphology resulting from an overall thinner film.

\section{Early Stage Deposition at Highest Speed}

From the results of section I it became apparent that the Re was influencing the microstructure and overall continuity of the Ag films. In order to further understand this, we investigated the early stages of deposition as well as film growth as a function of time utilizing a combination of SEM (Phenom ProX) and optical spectroscopy.

\section{Minute}

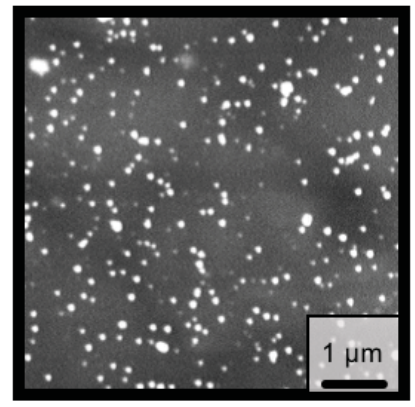

1.5 Minutes

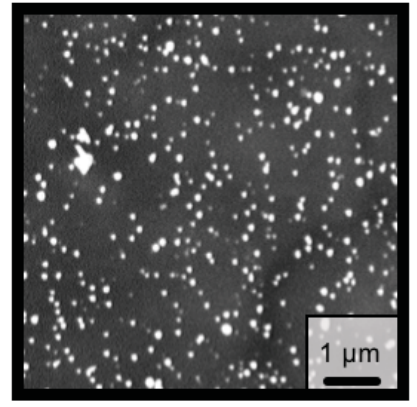

2 Minutes

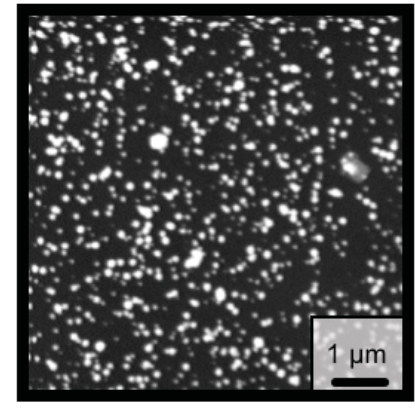

Figure 5. SEM images of Ag nanoparticles at $10 \mathrm{~cm}$ after 1, 1.5, and 2 minutes.

a. SEM investigation of Ag deposition with time: In Figure 5, SEM images taken following deposition for 1, 1.5, and 2 minutes of solution flowing at the highest $R e=300$ speed are shown. From the scale bar, it is evident that the Ag deposits at the earliest time are in the nanometer size scale. Further, increasing flow time leads to an increasing concentration of the number of these nanoparticles. At 3 minutes, evidence for agglomeration resulting in larger particles is also apparent. From this analysis, the high resistance of the $R e=300$ film measured in Figure 4(b) is supported by a deposition process that proceeds by deposition of discontinuous clusters of $\mathrm{Ag}$, thus making it harder to form a wellconnected Ag thin film microstructure.

b. Localized Surface Plasmon Resonance (LSPR) of Ag Nanoparticles (NPs): We also utilized optical spectroscopy to characterize the early stages of film deposition because Ag nanostructures show strong LSPR signal due to the conduction electrons. ${ }^{28}$ When light or electromagnetic waves interact with the Ag NPs, the electron cloud in the NPs oscillate collectively at a particular resonant frequency, giving rise to the LSPR peak. ${ }^{29}$ These resonances occur at specific wavelengths (or frequencies) that depend on particle size, shape, and the type of surrounding medium. ${ }^{30}$ Thus by observing the position of these resonances via optical spectroscopy, it is possible to determine information about the Ag nanos- 


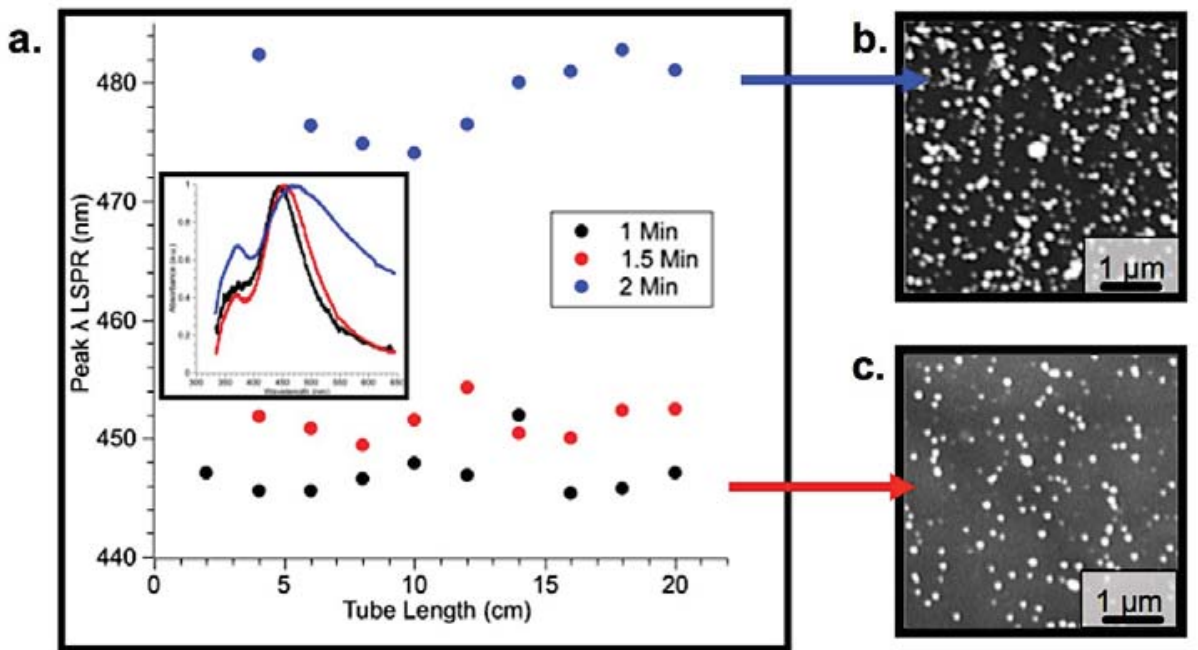

Figure 6. (a) Peak LSPR wavelengths as a function of tube length after 1, 1.5, and 2 minutes. (b) SEM image of nanoparticles after two minutes at $10 \mathrm{~cm}$ c. SEM image of nanoparticles after 1.5 minutes at $10 \mathrm{~cm}$.

tructures. In Figure 6(a) the wavelength of the LSPR peak measured at various locations along the length of the tube are plotted for the three early stage deposition times. The inset of Figure 6(a) shows typical absorbance curves from the middle of the tube for the three deposition times of 1, 1.5 and 2 mins. The LSPR wavelength corresponds to the intense peak visible around $450 \mathrm{~nm}$. From the results in Figure 6(a), the resonance wavelength appears to increase by a small amount in going from 1 to 1.5 minutes. From the SEM image in Figure 5, this increase is most likely due to a small increase in the average size of the Ag nanoparticles, which typically results in a red shift of the LSPR wavelength. More dramatic is the large red shift from the Ag deposited for 2 minutes, as seen by the blue dots in Figure 6(a). This large shift is most likely an indication of the substantial agglomeration happening in the Ag films, consistent with the SEM information shown in Figure 5. This result also suggests that LSPR could be used to monitor the deposition process in real-time in order to observe the transition from deposition of discrete islands of $\mathrm{Ag}$ to one where the agglomeration begins. Indeed, recently in-situ investigations have utilized Ag LSPR to monitor the deposition of thin films. ${ }^{31}$

\section{CONCLUSIONS}

In this paper, we explored the role of Reynolds number on the deposition of Ag thin film inside hollow cylindrical structure via the chemical bath deposition process. By utilizing a combination of scanning electron microscopy and electrical resistivity it was found that the uniformity of a film requires an optimal speed. More specifically, it was found that the Reynolds number of 203 provides a good balance between the deposition rate of the silver precipitates and the the formation of a film with fairly consistent film thickness throughout the length of a $22 \mathrm{~cm}$ hollow fiber with 0.8 $\mathrm{cm}$ inner diameter. The film quality was also verified by the overall low electrical resistance for this flow condition. Analysis of the early stage film deposition using SEM and localized surface plasmon resonance spectroscopy showed that the highest flow rate $(R e=300)$ produces segregated Ag film deposits at the early stage, likely resulting in the high resistivity of these films. Overall, these results show that controlling the flow rate can have substantial impact on the growth and ensuing morphology and physical properties of Ag thin films deposited inside hollow cylinders.

\section{ACKNOWLEDGMENTS}

This research was supported by NSF grant CBET-1349507 and TNSCORE grant NSF-EPS-10004083. This work was also supported by the Research and Instructional Strategies for Engineering Retention (RISER) at the University of Tennessee, Knoxville which is funded by the NSF through the Science, Technology, Engineering, and Mathematics Talent Expansion Program (STEP) award number 1068103. RK and HT acknowledge Oak Ridge National Laboratory CNMS grant CNMS2013-284 and University of Tennessee Department of Materials Science for SEM access. 


\section{REFERENCES}

1. Green, M. A., Emery, K., Hishikawa, Y., and Warta, W. (2009) Solar Cell Efficiency Tables (version 33), Progress in Photovoltaics: Research and Applications 17, 85-94.

2. Kalyanaraman, R., Duscher, G., Krishnan, V., Garcia, H., and Malasi, A. (2015) Hollow photovoltaic fiber, U.S. Patent No. 9,054,248.

3. Tachan, Z., Ruhle, S., and Zaban, A. (2010) Dye-sensitized solar tubes: A new solar cell design for efficient current collection and improved cell sealing, Sol Energ Mat Sol C, 94, 317-322.

4. Solyndra, Wholesale SOLAR, https://wrww.wholesalesolar.com/brands/solyndra, accessed 06/11/2018

5. Li, Y., Peterson, E. D., Huang, H., Wang, M., Xue, D., Nie, W., Zhou, W., \& Carroll, D. L. (2010) Tube-based geometries for organic photovoltaics, Appl Phys Lett 96, 243505-243508.

6. Gaudiana, R., Li, L., Chittibabu, K., Eckert, R., Montello, A., Montello, E., and Wormser, P. (2011) Photovoltaic fibers, US Patent No. 7,894,694.

7. Huang, H., Li, Y., Wang, M., Nie, W., Zhou, W., Peterson, E., Liu, J., Fang, G., and Carroll, D. L. (2011) Photovoltaic thermal solar energy collectors based on optical tubes, Sol Energy 85, 450-454.

8. Garnett, E. C. and Yang, P. (2008) Silicon nanowire radial p-n junction solar cells, J Am Chem Soc 130, 9224-9225.

9. Jung, J.-Y., Guo, Z., Jee, S.-W., Um, H.-D., Park, K.-T., Hyun, M. S., Yang, J. M., and Lee, J.-H. (2010) A waferscale Si wire solar cell using radial and bulk p-n junctions, Nanotechnology 21, 445303-445309.

10. Kelzenberg, M. D., Boettcher, S. W., Petykiewicz, J. A., Turner-Evans, D. B., Putnam, M. C., Warren, E. L., Spurgeon, J. M., Briggs, R. M., Lewis, N. S. and Atwater, H. A. (2010) Enhanced absorption and carrier collection in Si wire arrays for photovoltaic applications, Nat Mater 9, 239-244.

11. Kuo, C.-L., Wang, R.-C., Huang, J.-L., Liu, C.-P., Wang, C.-K., Chang, S.-P., Chu, W.-H., Wang, C.-H. \& Tu, C.H. (2009) The synthesis and electrical characterization of $\mathrm{Cu}(2) \mathrm{O} / \mathrm{Al}: \mathrm{ZnO}$ radial $\mathrm{p}-\mathrm{n}$ junction nanowire arrays, Nanotechnology 20, 365603-365610.

12. Yoon, H. P., Yuwen, Y. A., Kendrick, C. E., Barber, G. D., Podraza, N. J., Redwing, J. M., Mallouk, T. E., Wronski, C. R., and Mayer, T. S. (2010) Enhanced conversion efficiencies for pillar array solar cells fabricated from crystalline silicon with short minority carrier diffusion lengths, Appl Phys Lett 96, 213503-213506.

13. Dasgupta, P.K., Genfa, Z., Poruthoor, S.K., Caldwell, S., Dong, S. and Liu, S.Y. (1998) High-sensitivity gas sensors based on gas-permeable liquid core waveguides and long-path absorbance detection, Anal Chem 70, 4661-4669.

14. Eijkelenborg, M.A., Argyros, A., Barton, G., Bassett, I.M., Fellew, M., Henry, G., Issa, N.A., Large, M.C.J., Manos, S., Padden, W., Poladian, L. and Zagari, J. (2003) Recent progress in microstructured polymer optical fibre fabrication and characterisation, Opt Fiber Technol 9, 199-209.

15. Fink, Y., Ripin, D., Fan, S., Chen, C., Joannopoulos, J., and Thomas, E. (1999) Guiding optical light in air using an all-dielectric structure, J Lightwave Technol 17, 2039-2041.

16. Harrington, J. A. (2000) A review of IR transmitting, hollow waveguides, Fiber Integrated Opt 19, 211-227.

17. Johnson, S., Ibanescu, M.,Skorobogatiy, M., Weisberg, O., Engeness, T., Soljacic, M., Jacobs, S., Joannopoulos, J., and Fink, Y. (2001) Low-loss asymptotically single-mode propagation in large-core OmniGuide fibers, Opt Express 9, 748-779.

18. Kato, Y., Osawa, M., Miyagi, M., Abe, S., Aizawa, M., and Onodera, S. (1995) Loss characteristics of polymidecoated silver hollow glass wave-guides for the infrared, Electron Lett 31, 31-32.

19. Matsuura, Y. and Miyagi, M. (1998) Flexible hollow waveguides for delivery of excimer-laser light, Opt Lett 23, $1226-1228$.

20. Rabii, C. D., and Harrington, J. A. (1996) Optical properties of dual-core hollow waveguides, Appl Optics 35, 6249-625: 
21. Yan, M. and Mortensen, N. A. (2009) Hollow-core infrared fiber incorporating metal-wire metamaterial, Opt Express $17,14851-14864$.

22. Mohebbi, M., Fedosejevs, R., Gopal, V., and Harrington, J. (2002) Silver-coated hollow-glass waveguide for applications at $800 \mathrm{~nm}$, Appl Optics 41, 7031-7035.

23. Gopal, V., and Harrington, J. (2003) Deposition and characterization of metal sulfide dielectric coatings for hollow glass waveguides, Opt Express 11, 3182-3187.

24. Gopal, V. (2003) New dielectric coatings for low-loss hollow glass waveguides and bundles. Ph.D. Thesis, Rutgers, The state university of New Jersey, 52-54

25. Gopal, V., Harrington, J., Goren, A., and Gannot, I. (2004) Coherent hollow-core waveguide bundles for infrared imaging, Opt Eng 43, 1195-1199.

26. Mane, R. S. and Lokhande, C. D. (2000) Chemical deposition method for metal chalcogenide thin flms, Mater Chem Phys 65, 1-31.

27. Mayes, C., Schlichting, H., Krause, E., Oertel, H., and Gersten, K., (2003) Boundary-Layer Theory, Springer Berlin Heidelberg.

28. Lee, K. S. and El-Sayed, M. A. (2006) Gold and silver nanoparticles in sensing and imaging: Sensitivity of plasmon response to size, shape, and metal composition, J Phys Chem B 10, 19220-19225.

29. Hao, E. and Schatz, G. C. (2004) Electromagnetic Ỵelds around silver nanoparticles and dimers, J Chem Phys 120, 357-366.

30. Haes, A. J. and Van Duyne, R. P. (2002) A nanoscale optical biosensor: Sensitivity and selectivity of an approach based on the localized surface plasmon resonance spectroscopy of triangular silver nanoparticles, JAm Chem Soc 124, 10596-10604.

31. Taz, H. and Ruther, R. and Malasi, A. and Yadavali, S. and Carr, C. and Nanda, J. and Kalyanaraman, R. (2015) In situ localized surface plasmon resonance (lspr) spectroscopy to investigate kinetics of chemical bath deposition of cds thin films, J Phys Chem C 119, 5033-5039.

\section{ABOUT THE STUDENT AUTHORS}

Mikayla Ehrsam graduated from the University of Tennessee in May of 2018 with a Bachelor of Science in Mechanical Engineering and a minor in Reliability and Maintainability Engineering. She is currently working for Southern Nuclear. Humaira Taz is a PhD student studying Energy Science and Engineering at the University of Tennessee's Bredesen Center. Connor Carr graduated from the University of Tennessee in May of 2016 with a Bachelor of Science in Materials Science and Engineering. He is currently pursuing a PhD in Materials Science and Engineering at Northwestern University. Abhinav Malasi completed his PhD in Chemical Engineering at the University of Tennessee in May of 2016 and is currently looking for academic positions while taking time off in Europe.

\section{PRESS SUMMARY}

In order to construct such a solar device in a hollow polymer cylinder, one must be able to use chemical bath deposition to deposit homogeneous films with the appropriate microstructure. The objective of this project was to deposit a smooth, homogeneous silver film inside a hollow cylindrical structure using varying flow rates. The early stage film growth for very short deposition times was observed by the localized surface plasmon resonance of the silver nanoclusters via absorption spectra along the length of the tube. For longer deposition times, silver films formed and were analyzed for their morphology, thickness, roughness, and resistance using a combination of optical microscopy, scanning electron microscopy, and two-probe conductivity. The findings from this study showed that deposition under flow with different Reynolds numbers had a strong influence on the early stage and final morphology, and resistance of the deposited films. 